\title{
Targeted Imaging
}

National Cancer Institute

\section{Source}

National Cancer Institute. Targeted Imaging. NCI Thesaurus. Code C154910.

Imaging that uses tagged molecules that are specific for a target molecule or biomarker. 